\title{
UPDATED CONSTRAINTS ON HEAVY RESONANCES USING THE RUN 2 DATA AT LHC
}

\author{
A.A. Pankov ${ }^{1,2}$, I.A. Serenkova ${ }^{1}$, V.A. Bednyakov ${ }^{2}$ \\ ${ }^{1}$ Abdus Salam ICTP Affiliated Centre at Gomel State Technical University, \\ Gomel 246746, Belarus, pankov@ictp.it \\ 2 Joint Institute for Nuclear Research, JINR, Dubna 141980, Russia
}

\begin{abstract}
The full ATLAS and CMS Run 2 data set at the Large Hadron Collider (LHC) with timeintegrated luminosity of $139 \mathrm{fb}^{-1}$ and $137 \mathrm{fb}^{-1}$, respectively, in the diboson channel is used to probe benchmark models with extended gauge sectors: the $E_{6}$-motivated Grand Unification models, the left-right symmetric LR and the sequential standard model. These all predict neutral $Z^{\prime}$ and charged $W^{\prime}$ vector bosons, decaying into lepton or electroweak gauge boson pairs. We present constraints on the parameter space of the $Z^{\prime}$ and $W^{\prime}$ and compare them to those obtained from the previous analyses performed with LHC data collected at 7 and $8 \mathrm{TeV}$ in Run 1 as well as at $13 \mathrm{TeV}$ in Run 2 at time-integrated luminosity of $36.1 \mathrm{fb}^{-1}$. We show that proton-proton collision data at $\sqrt{s}=13 \mathrm{TeV}$ collected by the ATLAS and the CMS experiments allow to set the most stringent bounds to date on $Z-Z^{\prime}$ and $W-W^{\prime}$ mixing.
\end{abstract}

AНОТАЦЯ. Повний набір даних ATLAS i CMS Run 2 на Великому адронному колайдері (LHC) 3 інтегрованою за часом світністю 139 фб ${ }^{-1}$ і 137 фб -1 , відповідно, у дибозонному каналі використовується для тестування еталонних моделей 3 розширеними калібрувальними

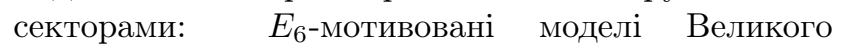
об'єднання, ліво-право симетрична LR та послідовна стандартна модель. Всі вони передбачають нейтральні $Z^{\prime}$ і заряджені векторні $W^{\prime}$-бозони, що розпадаються на лептонні або електрослабкі калібрувальні бозони. Ми представляємо обмеження на простір параметрів $Z^{\prime}$ і $W^{\prime}$ і порівнюємо їх з тими, які були отримані 3 попередніх аналізів, виконаними 3 даними LHC, зібраними при 7 i 8 TeB в Run 1, a також при $13 \mathrm{TeB}$ в Run 2 при інтегрованій за часом світністю 36.1 фб $^{-1}$. Ми показуємо, що дані про зіткнення протонів при $\sqrt{s}=13 \mathrm{TeB}$, зібрані за допомогою експериментів ATLAS i CMS, дозволяють встановити найсуворіші на сьогоднішній день обмеження для $Z-Z^{\prime}$ i $W$ - $W^{\prime}$ змішування.

Keywords: Physics beyond the Standard model, new gauge $Z^{\prime}$ and $W^{\prime}$ bosons, Large Hadron Collider.

\section{Introduction}

Searches for new heavy resonances with their subsequent decay into a final state consisting of a pair of fermions or vector bosons are one of the major goals of the ATLAS and CMS physics programmes at the LHC at CERN. Possible candidates of physics scenarios beyond the Standard Model (SM) are charged and neutral heavy gauge bosons. In the simplest models these particles are considered copies of the SM $W$ and $Z$ bosons and are commonly referred to as $W^{\prime}$ and $Z^{\prime}$ bosons (for review and original references, see Zyla et. al., 2020).

At the LHC, such heavy $W^{\prime}$ and $Z^{\prime}$ bosons could be observed through their single production as $s$-channel resonances with subsequent leptonic decays

$$
p p \rightarrow W^{\prime} X \rightarrow \ell \nu X
$$

and

$$
p p \rightarrow Z^{\prime} X \rightarrow \ell \nu X
$$

respectively, where in what follows, $\ell=e, \mu$ unless otherwise stated. The dilepton final states are considered the golden channels for the discovery of the heavy resonances, $Z^{\prime}$ and $W^{\prime}$, thanks to the minimal backgrounds from the SM. The production of $W^{\prime}$ and $Z^{\prime}$ bosons at hadron colliders is expected to be dominated by the process $q \bar{q}^{\prime} / q \bar{q} \rightarrow W^{\prime} / Z^{\prime}$. Leptonic final states provide a low-background and efficient experimental signature that results in excellent sensitivity to new phenomena at the LHC.

In the simplest models such as the Sequential Standard Model (SSM) (Zyla et. al., 2020) new neutral $Z_{\mathrm{SSM}}^{\prime}$ and charged $W_{\mathrm{SSM}}^{\prime}$ bosons have couplings to fermions that are identical to those of the SM $Z$ and $W$ bosons, but for which the trilinear couplings $Z^{\prime} W W$ and $W^{\prime} W Z$ are absent. The SSM has been used as a reference for experimental $Z^{\prime}$ and $W^{\prime}$ searches for decades, the results can be re-interpreted in the context of other models, it is therefore useful for compar- 
ing the sensitivity of different experiments. Another class of models considered here are those inspired by Grand Unified Theories (GUT), which are motivated by gauge unification or a restoration of the left-right symmetry violated by the weak interaction. Examples considered in this paper include the $Z^{\prime}$ bosons of the $E_{6}$-motivated theories; and high-mass neutral bosons of the left-right (LR) symmetric extensions of the SM, based on the $S U(2)_{L} \otimes S U(2)_{R} \otimes U(1)_{B-L}$ gauge group, where $B-L$ refers to the difference between baryon and lepton numbers.

The data we consider were collected with the ATLAS and CMS detectors during the 2015-2018 running period of the LHC, referred to as Run 2 and corresponding to a time-integrated luminosity of $\sim 140 \mathrm{fb}^{-1}$. The ATLAS and CMS collaborations set a $95 \%$ confidence level (CL) lower limit on the $Z^{\prime}$ mass of $\sim 4.6 \mathrm{TeV}-$ 5.2 TeV depending on the model, and 6.0 TeV for the $W_{\mathrm{SSM}}^{\prime}$.

Alternative $Z^{\prime}$ and $W^{\prime}$ search channels are the diboson reactions

$$
p p \rightarrow Z^{\prime} X \rightarrow W W X
$$

and

$$
p p \rightarrow W^{\prime} X \rightarrow W Z X
$$

The study of gauge boson pair production can be used as a probe for new phenomena beyond the SM. Specifically, in contrast to the DY processes (2) and (1) diboson reactions are not the primary discovery channels, but can help to understand the origin of new gauge bosons. Here, we concentrate on the LHC potential for precision determination of $Z-Z^{\prime}$ and $W-W^{\prime}$ mixing angles, referred to as $\xi_{Z-Z^{\prime}}$ and $\xi_{W-W^{\prime}}$ (for details of the analysis and original references, see Osland et al., 2021).

Previous analyses of the $Z-Z^{\prime}$ and $W-W^{\prime}$ mixing (Osland et al., 2017; Bobovnikov et al., 2018; Serenkova et al., 2019) were carried out using the diboson and dilepton production data sets corresponding to the time-integrated luminosity of $\sim 36$ $\mathrm{fb}^{-1}$ collected in 2015 and 2016 with the ATLAS and CMS collaborations at $\sqrt{s}=13 \mathrm{TeV}$. The results of the present analysis benefit from the increased size of the data sample, now amounting to an integrated luminosity of $\sim 140 \mathrm{fb}^{-1}$ recorded by the ATLAS and CMS detectors in Run 2, almost four times larger than what was available for the previous study. Thus, our present analysis is complementary to the previous studies.

\section{2. $Z^{\prime}$ hadronic production and decay}

The $Z^{\prime}$ production and subsequent decay into $W W$ in proton-proton collisions occurs via quark-antiquark annihilation in the $s$-channel. The cross section of process (3) can at the LHC be observed through resonant pair production of gauge bosons $W W$. Using the narrow width approximation (NWA), one can factorize the process (3) into the $Z^{\prime}$ production and its subsequent decay,

$$
\sigma\left(p p \rightarrow Z^{\prime} X\right) \times \operatorname{BR}\left(Z^{\prime} \rightarrow W W\right)
$$

Here, $\sigma\left(p p \rightarrow Z^{\prime} X\right)$ is the total (theoretical) $Z^{\prime}$ production cross section and $\operatorname{BR}\left(Z^{\prime} \rightarrow W W\right)=\Gamma_{Z^{\prime}}^{W W} / \Gamma_{Z^{\prime}}$ with $\Gamma_{Z^{\prime}}$ the total width of the $Z^{\prime}$. "Narrow" refers to the assumption that the natural width of the resonance is smaller than the typical experimental resolution of $5 \%$ of its mass. This is valid for a large fraction of the parameter space of the considered models.

In the calculation of the total width $\Gamma_{Z^{\prime}}$ we consider the following channels: $Z^{\prime} \rightarrow f \bar{f}, W^{+} W^{-}$, and $Z H$, where $H$ is the SM Higgs boson and $f$ refers to the SM fermions $(f=l, \nu, q)$. The total width $\Gamma_{Z^{\prime}}$ of the $Z^{\prime}$ boson can then be written as follows:

$$
\Gamma_{Z^{\prime}}=\sum_{f} \Gamma_{Z^{\prime}}^{f f}+\Gamma_{Z^{\prime}}^{W W}+\Gamma_{Z^{\prime}}^{Z H} .
$$

The two last terms, which are often neglected in studies at low and moderate values of $M_{Z^{\prime}}$, are due to $Z-Z^{\prime}$ mixing. However, this is no the case for heavier resonances.

For a fixed mixing factor $\xi_{Z-Z^{\prime}}$ and at large $M_{Z^{\prime}}$ where $\Gamma_{Z^{\prime}}^{W W}$ dominates over $\sum_{f} \Gamma_{Z^{\prime}}^{f f}$ the total width increases rapidly with the mass $M_{Z^{\prime}}$ because of the quintic dependence of the $W^{+} W^{-}$mode on the $Z^{\prime}$ mass. In this case, the $W^{+} W^{-}$mode (together with $Z^{\prime} \rightarrow Z H$ ) becomes dominant and $\operatorname{BR}\left(Z^{\prime} \rightarrow W^{+} W^{-}\right) \rightarrow 0.5$ (this value arises from the assumption $\Gamma_{Z^{\prime}}^{Z H}=\Gamma_{Z^{\prime}}^{W}$ ), while the fermionic decay channels $\left(\Gamma_{Z^{\prime}}^{f f} \propto M_{Z^{\prime}}\right)$ are increasingly suppressed.

In Fig. 1, we consider the full ATLAS and CMS Run 2 data set of time integrated luminosity of 137 $\mathrm{fb}^{-1}$ and $139 \mathrm{fb}^{-1}$, respectively, and show the observed 95\% C.L. upper limits on the production cross section times the branching fraction, $\sigma_{95 \%} \times \mathrm{BR}\left(Z^{\prime} \rightarrow\right.$ $\left.W^{+} W^{-}\right)$, as a function of the $Z^{\prime}$ mass, obtained from the semileptonic final states. This allows for a comparison of the sensitivities of the data to mixing parameters and new gauge boson mass.

Then, in Fig. 2 we compute the LHC production cross section multiplied by the branching ratio into two $W$ bosons, $\sigma\left(p p \rightarrow Z_{\mathrm{EGM}}^{\prime} X\right) \times \mathrm{BR}\left(Z_{\mathrm{EGM}}^{\prime} \rightarrow W^{+} W^{-}\right)$, as a function of the two parameters $\left(M_{Z^{\prime}}, \xi_{Z-Z^{\prime}}\right)$, and compare it with the limits established by the ATLAS experiment, $\sigma_{95 \%} \times \mathrm{BR}\left(Z^{\prime} \rightarrow W^{+} W^{-}\right)$.

Different bounds on the $Z^{\prime}$ parameter space are collected in Fig. 2 for the $Z_{\mathrm{EGM}}^{\prime}$ model, showing that at high masses, the limits on $\xi_{Z-Z^{\prime}}$ obtained from the full Run 2 data set collected at $\sqrt{s}=13 \mathrm{TeV}$ and recorded by the ATLAS and CMS detectors are substantially stronger than that derived from the global analysis of the precision electroweak data, which is also displayed. 


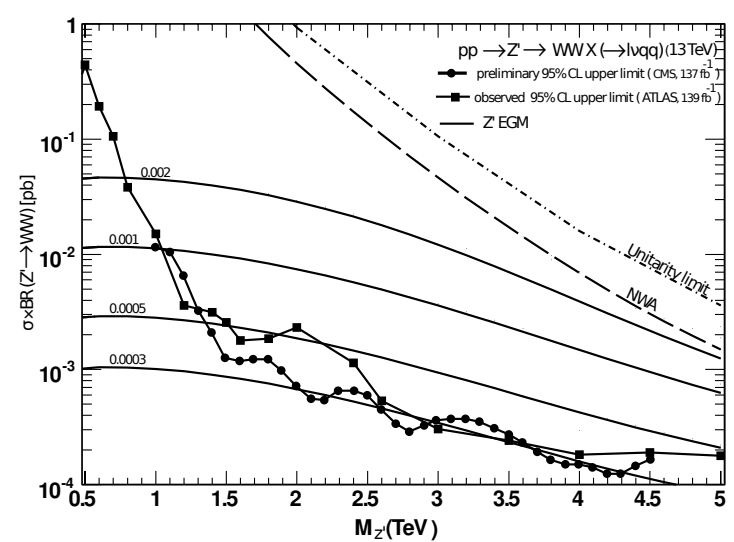

Figure 1: Expected 95\% C.L. upper limits on the production cross section times the branching fraction for $Z_{\mathrm{EGM}}^{\prime} \rightarrow W^{+} W^{-}$as a function of $Z^{\prime}$ mass, $M_{Z^{\prime}}$, at time-integrated luminosity of $137 \mathrm{fb}^{-1}$ (CMS) and 139 $\mathrm{fb}^{-1}$ (ATLAS). Theoretical production cross sections $\sigma \cdot B R\left(Z_{\mathrm{EGM}}^{\prime} \rightarrow W^{+} W^{-}\right)$are given by solid curves. Labels attached to the curves for the $Z_{\mathrm{EGM}}^{\prime}$ cross section correspond to the considered mixing factor $\xi_{Z-Z^{\prime}}$.

Analogous analysis can be made for other benchmark models of $Z^{\prime}$ bosons under study with extended gauge sector. It turns out that the current limits on the $Z-Z^{\prime}$ mixing parameters, $\xi_{Z-Z^{\prime}}$, from the EW precision data are generally weaker than those from the LHC limits obtained in Run 2 at $13 \mathrm{TeV}$ with time-integrated luminosity of $\mathcal{L}_{\text {int }} \sim 140 \mathrm{fb}^{-1}$. In fact, the latter improve the EW limits by approximately one order of magnitude, depending on the resonance mass.

\section{3. $W^{\prime}$ hadronic production and decay}

We consider the simplest EGM model which predicts charged heavy gauge bosons. The analysis of $W-W^{\prime}$ mixing in diboson and dilepton pair production which will be performed below is quite analogous to that carried out in previous sections for $Z-Z^{\prime}$ mixing. At lowest order in the EGM, $W^{\prime}$ production and decay into $W Z$ in proton-proton collisions occurs through quark-antiquark annihilation in the $s$-channel. Using the NWA, one can factorize the process (4) into the $W^{\prime}$ production and the $W^{\prime}$ decay,

$$
\sigma\left(p p \rightarrow W^{\prime} X\right) \times \mathrm{BR}\left(W^{\prime} \rightarrow W Z\right) .
$$

Here, $\sigma\left(p p \rightarrow W^{\prime} X\right)$ is the total (theoretical) $W^{\prime}$ production cross section and $\operatorname{BR}\left(W^{\prime} \rightarrow W Z\right)=\Gamma_{W^{\prime}}^{W Z} / \Gamma_{W^{\prime}}$ with $\Gamma_{W^{\prime}}$ the total width of the $W^{\prime}$.

In the EGM the $W^{\prime}$ bosons can decay into SM fermions, gauge bosons $(W Z)$, or $W H$. In the calculation of the total width $\Gamma_{W^{\prime}}$ we consider the following

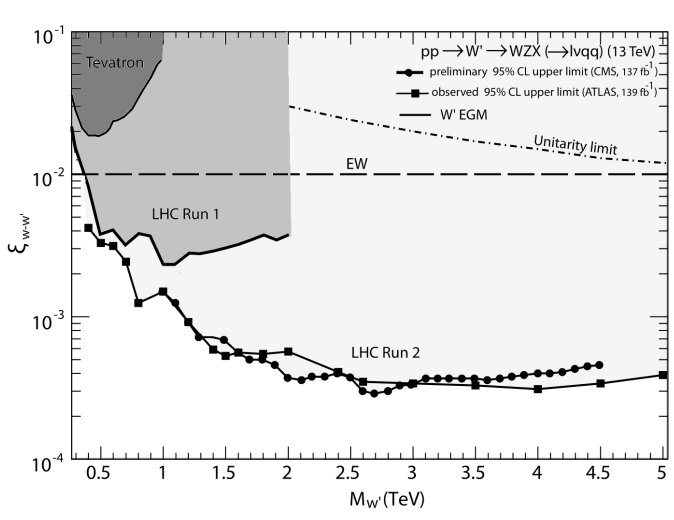

Figure 2: Expected 95\% C.L. exclusion regions in the two-dimensional $\left(M_{Z^{\prime}}, \xi_{Z-Z^{\prime}}\right)$ plane obtained after incorporating direct search constraints from the CDF and D0 collaborations which are referred to as Tevatron in $p \bar{p} \rightarrow W^{+} W^{-} X$ as well as those derived from the LHC measurement of $p p \rightarrow W W X$ in Run 1 and 13 $\mathrm{TeV}$ from diboson $Z^{\prime} \rightarrow W W$ production in semileptonic final states using the partial and full Run 2 ATLAS and CMS data set. Also shown is the exclusion from the precision electroweak $(\mathrm{EW})$ data. The uninarity limit is shown as a dot-dashed curve.

channels: $W^{\prime} \rightarrow f \bar{f}^{\prime}, W Z$, and $W H$, where $f$ is a SM fermion $(f=\ell, \nu, q)$. Only left-handed neutrinos are considered, possible right-handed neutrinos are assumed to be kinematically unavailable as final states. Also, like for the $Z^{\prime}$ case, we shall ignore the couplings to other beyond-SM particles such as SUSY partners and exotic fermions. As a result, the total decay width of the $W^{\prime}$ boson is taken to be

$$
\Gamma_{W^{\prime}}=\sum_{f} \Gamma_{W^{\prime}}^{f \bar{f}^{\prime}}+\Gamma_{W^{\prime}}^{W Z}+\Gamma_{W^{\prime}}^{W H}
$$

Like for the $Z^{\prime}$ case, the presence of the last two decay channels, which are often neglected at low and moderate values of $M_{W^{\prime}}$, is due to $W-W^{\prime}$ mixing which is constrained to be tiny. In particular, for the range of $M_{W^{\prime}}$ values below $\sim 1.0-1.5 \mathrm{TeV}$, the dependence of $\Gamma_{W^{\prime}}$ on the values of $\xi_{W-W^{\prime}}$ (within its allowed range) induced by $\Gamma_{W^{\prime}}^{W Z}$ and $\Gamma_{W^{\prime}}^{W H}$ is unimportant because $\sum_{f} \Gamma_{W^{\prime}}^{f \bar{f}^{\prime}}$ dominates over the diboson partial widths. Therefore, in this mass range, one can approximate the total width as $\Gamma_{W^{\prime}} \approx \sum_{f} \Gamma_{W^{\prime}}^{f \bar{f}^{\prime}}=3.5 \% \times M_{W^{\prime}}$ (Serenkova et al.: 2019), where the sum runs over SM fermions only.

For heavier $W^{\prime}$ bosons, the diboson decay channels, $W Z$ and $W H$, start to play an important role, and we are no longer able to ignore them. To be specific, in analogy with the $Z^{\prime}$ case, we assume that both partial widths are comparable, $\Gamma_{W^{\prime}}^{W H} \simeq \Gamma_{W^{\prime}}^{W Z}$ for heavy $M_{W^{\prime}}$, as required by the Equivalence theorem (Serenkova et al.: 2019). 


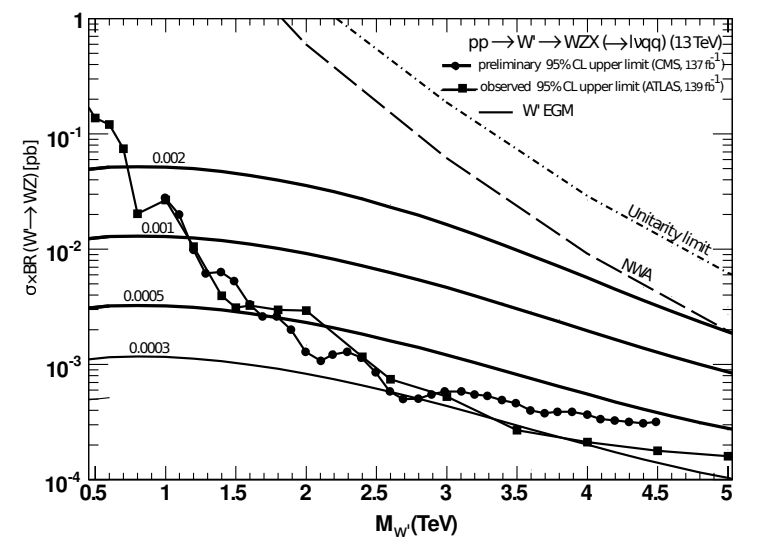

Figure 3: Expected 95\% C.L. upper limits on the production cross section times the branching fraction for $W^{\prime} \rightarrow W Z$ as a function of $W^{\prime}$ mass, $M_{W^{\prime}}$. Theoretical production cross sections $\sigma \cdot B R\left(W_{\mathrm{EGM}}^{\prime} \rightarrow W Z\right)$ for $W_{\mathrm{EGM}}^{\prime}$ are given by thin solid curves. Labels attached to the curves for the $W_{\mathrm{EGM}}^{\prime}$ cross section correspond to the considered mixing factor $\xi_{W-W^{\prime}}$.

For a fixed mixing factor $\xi_{W-W^{\prime}}$ and at large $M_{W^{\prime}}$, the total width increases rapidly with the $W^{\prime}$ mass because of the quintic dependence of the $W Z$ mode on the $W^{\prime}$ mass $\Gamma_{W^{\prime}}^{W Z} \propto M_{W^{\prime}}\left[M_{W^{\prime}}^{4} /\left(M_{W}^{2} M_{Z}^{2}\right)\right]$, corresponding to the production of longitudinally polarized $W$ and $Z$ in the channel $W^{\prime} \rightarrow W_{L} Z_{L}$. In this case, the $W Z$ mode (as well as $W H$ ) becomes dominant and $\operatorname{BR}\left(W^{\prime} \rightarrow W Z\right) \rightarrow 0.5$, while the fermionic decay channels, $\sum_{f} \Gamma_{W^{\prime}}^{f \bar{f}^{\prime}} \propto M_{W^{\prime}}$, are increasingly suppressed.

In Fig. 3, we show the observed 95\% C.L. upper limits on the production cross section times the branching fraction, $\sigma_{95 \%} \times \mathrm{BR}\left(W^{\prime} \rightarrow W Z\right)$, as a function of the $W^{\prime}$ mass.

Then, for $W^{\prime}$ we compute the LHC theoretical production cross section multiplied by the branching ratio into $W Z$ bosons, $\sigma\left(p p \rightarrow W^{\prime} X\right) \times \mathrm{BR}\left(W^{\prime} \rightarrow W Z\right)$, as a function of the two parameters $\left(M_{W^{\prime}}, \xi_{W-W^{\prime}}\right)$, and compare it with the limits established by the ATLAS and CMS experiments, $\sigma_{95 \%} \times \mathrm{BR}\left(W^{\prime} \rightarrow W Z\right)$.

The area below the long-dashed curve labelled "NWA" corresponds to the region where the $W^{\prime}$ resonance width is predicted to be less than $5 \%$ of its mass, corresponding to the best detector resolution of the searches, where the narrow-width assumption is satisfied. We also show a curve labelled "Unitarity limit" that corresponds to the unitarity bound and references therein). It was shown that the saturation of unitarity in the elastic scattering $W^{ \pm} Z \rightarrow W^{ \pm} Z$ leads to the constraint $\left(g_{W^{\prime} W Z}\right)_{\max }=g_{W W Z} \cdot M_{Z}^{2} /\left(\sqrt{3} M_{W^{\prime}} M_{W}\right)$ that was adopted in plotting this bound. The constraint was obtained under the assumption that the couplings of the $W^{\prime}$ to quarks and to gauge bosons

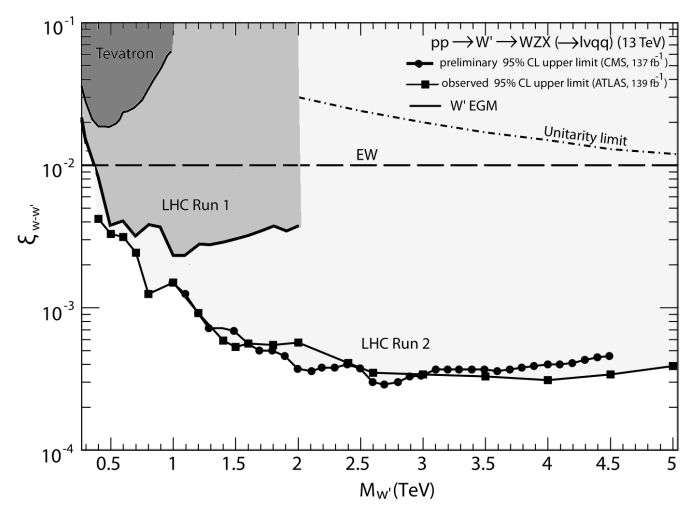

Figure 4: 95\% C.L. exclusion regions in the twodimensional $\left(M_{W^{\prime}}, \xi_{W-W^{\prime}}\right)$ plane obtained from the precision electroweak data (horizontal dashed straight line labeled "EW"), the direct search constraints at the Tevatron in $p \bar{p} \rightarrow W Z X$ as well as from the LHC measurement of $p p \rightarrow W Z X$ at $7 \mathrm{TeV}$ and $8 \mathrm{TeV}$ (Run 1) and at $13 \mathrm{TeV}$ from diboson $W^{\prime} \rightarrow W Z$ production using full Run 2 ATLAS and CMS data set. The uninarity limit is shown as a dot-dashed curve.

have the same Lorentz structure as those of the SM but with rescaled strength.

The theoretical curves for the cross sections $\sigma(p p \rightarrow$ $\left.W^{\prime} X\right) \times \mathrm{BR}\left(W^{\prime} \rightarrow W Z\right)$, in descending order, correspond to values of the $W-W^{\prime}$ mixing factor $\xi_{W-W^{\prime}}$ from 0.01 to 0.0003 . The intersection points of the measured upper limits on the production cross section with these theoretical cross sections for various values of $\xi_{W-W^{\prime}}$ give the corresponding lower bounds on $\left(M_{W^{\prime}}, \xi_{W-W^{\prime}}\right)$, displayed in Fig. 4.

\section{Concluding remarks}

Exploration of the diboson, $W W$ and $W Z$, and dilepton, $\ell \ell$ and $\ell \nu$, production at the LHC with the $13 \mathrm{TeV}$ data set allows to place stringent constraints on the $Z-Z^{\prime}$ and $W-W^{\prime}$ mixing parameters as well as on the $Z^{\prime}$ and $W^{\prime}$ masses for benchmark extended models, respectively. We derived such limits by using the full ATLAS and CMS Run 2 data set recorded at the CERN LHC, with integrated luminosity of $139 \mathrm{fb}^{-1}$ and $137 \mathrm{fb}^{-1}$, respectively.

By comparing the experimental limits to the theoretical predictions for the total cross section of the $Z^{\prime}$ and $W^{\prime}$ resonant production and their subsequent decays into $W W$ or $W Z$ pairs, we show that the derived constraints on the mixing parameters, $\xi_{Z-Z^{\prime}}$ and $\xi_{W-W^{\prime}}$, are substantially improved with respect to those obtained from the global analysis of low energy electroweak data, as well as compared to the diboson 
production study performed at the Tevatron, and to those based on the LHC Run 1 as well as at $13 \mathrm{TeV}$ in Run 2 at time-integrated luminosity of $\sim 36 \mathrm{fb}^{-1}$ and are the most stringent bounds to date. Further constraining of this mixing can be achieved from the analysis of data to be collected in Run 3 as well as at the next options of hadron colliders such as HL-LHC and HE-LHC.

Acknowledgements. This research has been partially supported by the Abdus Salam ICTP (TRIL Programme) and the Belarusian Republican Foundation for Fundamental Research.

\section{References}

Zyla P.A. et al.: 2020, Review of Particle Physics, PTEP 2020, No.8, $083 \mathrm{C} 01$.

Osland P. et al.: 2021 Phys. Rev. D, 103, 053009.

Osland P. et al.: 2017, Phys. Rev. D, 96, No. 5, 055040 .

Bobovnikov et al.: 2018 Phys. Rev. D, 98, 095029.

Serenkova et al.: 2019, Phys. Rev. D, 100,1, 015007.

Pankov A. A. et al. Eur. Phys. J. C, 80, 6, 503. 